\title{
Innovatives Kopfkissen
}

\section{Optimale Unterstützung der Halswirbelsäule im Schlaf}

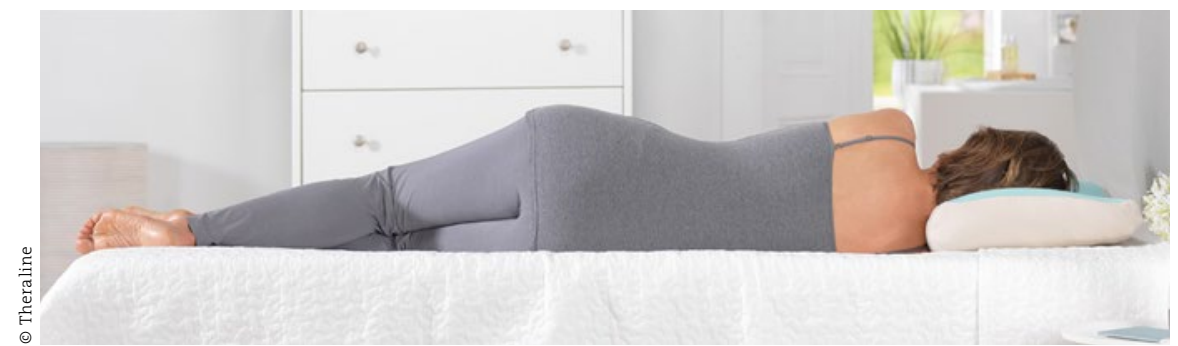

» Nackenprobleme, Rücken- oder Kopfschmerzen und Verspannungen - mit diesen Problemen haben immer mehr Menschen im Alltag zu kämpfen. Eine ergonomische Liegeposition und damit auch ein erholsamerer Schlaf mit Hilfe eines therapeutischen Kopfkissens können diese Symptome reduzieren. In der Regel ist das klassische Kopfkissen noch immer $80 \times 80 \mathrm{~cm}$ groß und mit Federn oder Polyester gefüllt. Diesen Varianten fehlt allerdings die stützende Funktion. Viele Kissenlösungen, die gezielt für nacken- und wirbelsäulenschonendes Liegen hergestellt werden, bestehen hingegen aus reinem Schaumstoff. Doch fehlt diesen Kissen aus Monomaterial die Fähigkeit, sich schnell anzupassen, da es träge auf Körperwärme reagiert. Dadurch stellt sich das Material nicht schnell genug darauf ein, wenn von der Rücken- in die
Seitenschlafposition gewechselt wird. Zudem werden diese Kissen häufig als zu hart oder zu weich empfunden. Weitere Aspekte, die kaum ein Modell erfüllt, sind die Kissengröße und -höhe. Dieser Faktor ist wichtig für die Unterstützung des Dreiecks zwischen Kopf, Hals und Schulter in der Seitenlage. Wird dieser Bereich nicht ausgefüllt, sinkt die Halswirbelsäule ab, ist er dagegen $\mathrm{zu}$ hoch oder stark gepolstert, entsteht eine Überstreckung. All diese Einflüsse können zu Nacken- oder Kopfschmerzen am Morgen führen. Doch was genau an der Schlafposition zu än-

\section{Fließende Stützfunktion}

Gemäß ganzheitlichen Therapieansätzen hat Theraline ein Kopfkissen entwickelt, das unterschiedliche Kompodern ist, wissen Patienten oft nicht. nenten miteinander verbindet. PEARLfusion kombiniert eine $4 \mathrm{~cm}$ dünne Schicht viskoelastischen Schaums mit Millionen feiner EPS-Mikroperlen aus Polystyrol. Bei der Herstellung durchlaufen diese sandfeinen Perlen ein spezielles Verfahren, damit sie sich bei der Nutzung später geräuschlos und fließend bei den nächtlichen Bewegungen verhalten. Der Füllkörper ist dabei in eine spezielle Keilform genäht und mit Abnähern versehen. Auf diese Weise fließen die Perlen stets in festgelegten Bahnen. Wie alle Kissen von Theraline lässt sich auch diese Innovation ganz einfach in der Höhe anpassen, indem die Perlen nach Bedarf aus dem Kissen entnommen oder hinzugefügt werden können. Ab sofort legt Theraline deshalb eine gut durchdachte Nachfüllpackung mit 2 Liter Mikroperlen bei.

Das Kissen ist in drei unterschiedlichen Höhen verfügbar $(10 \mathrm{~cm}, 12 \mathrm{~cm}$ und $14 \mathrm{~cm}$ ). Die Materialien sind für Allergiker geeignet und alle Komponenten, mit Ausnahme des Schaumstoffkerns, bei 60 Grad waschbar. PEARLfusion ist sowohl im stationären Fachhandel als auch im Online-Handel erhältlich.

\section{www.theraline.de}

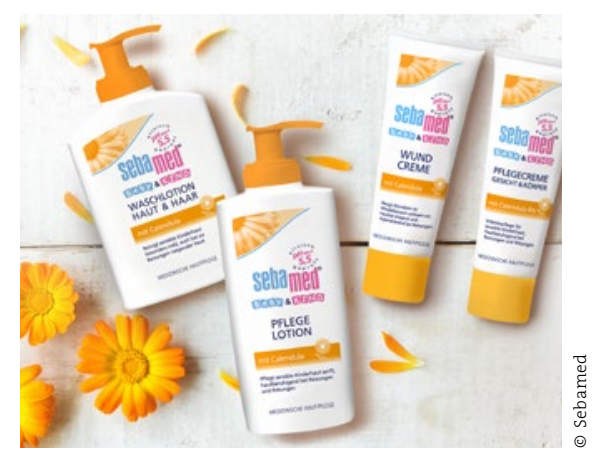

Verträglichkeit ausgezeichnet und kann auch therapiebegleitend bei Neurodermitis eingesetzt werden. Die sebamed Baby \& Kind mit Calendula Serie besteht aus Waschlotion, Pflegelotion, Pflegecreme und Wundcreme.

www.sebamed.de 